\title{
Intelligent Life Prediction of Thermal Barrier Coating for Aero Engine Blades
}

\author{
Ruipeng Gao ${ }^{1}$, Wei Mao ${ }^{1}$, Yiran Wang ${ }^{2}{ }^{\oplus}$, Shanshan Fan ${ }^{1}$ and Wei Shao ${ }^{1, *}$ \\ 1 School of Mechanical and Precision Instrument Engineering, Xi'an University of Technology, \\ Xi'an 710048, China; vessjessy@xaut.edu.cn (R.G.); m15291587080@163.com (W.M.); \\ 18309299206@163.com (S.F.) \\ 2 School of Materials Science and Engineering, Xi'an Jiaotong University, Xi'an 710049, China; \\ wangyiran@xjtu.edu.cn \\ * Correspondence: swlxm@xaut.edu.cn; Tel.: +86-029-82312863
}

Citation: Gao, R.; Mao, W.; Wang, Y.; Fan, S.; Shao, W. Intelligent Life Prediction of Thermal Barrier Coating for Aero Engine Blades. Coatings 2021, 11, 890. https://doi.org/10.3390/ coatings 11080890

Academic Editor: Narottam Bansal

Received: 8 June 2021

Accepted: 21 July 2021

Published: 26 July 2021

Publisher's Note: MDPI stays neutral with regard to jurisdictional claims in published maps and institutional affiliations.

Copyright: (c) 2021 by the authors. Licensee MDPI, Basel, Switzerland. This article is an open access article distributed under the terms and conditions of the Creative Commons Attribution (CC BY) license (https:// creativecommons.org/licenses/by/ $4.0 /)$.

\begin{abstract}
The existing methods for thermal barrier coating (TBC) life prediction rely mainly on experience and formula derivation and are inefficient and inaccurate. By introducing deep learning into TBC life analyses, a convolutional neural network $(\mathrm{CNN})$ is used to extract the TBC interface morphology and analyze its life information, which can achieve a high-efficiency accurate judgment of the TBC life. In this thesis, an Adap-Alex algorithm is proposed to overcome the problems related to the large training time, over-fitting, and low accuracy in the existing CNN training of TBC images with complex tissue morphologies. The method adjusts the receptive field size, stride length, and other parameter settings and combines training epochs with a sigmoid function to realize adaptive pooling. TBC data are obtained by thermal vibration experiments, a TBC dataset is constructed, and then the Adap-Alex algorithm is used to analyze the generated TBC dataset. The average training time of the Adap-Alex method is significantly smaller than those of VGG-Net and Alex-Net by 125 and $685 \mathrm{~s}$, respectively. For a fixed number of thermal vibrations, the test accuracy of the Adap-Alex algorithm is higher than those of Alex-Net and VGG-Net, which facilitates the TBC identification. When the number of thermal vibrations is 300 , the accuracy reaches $93 \%$, and the performance is highest.
\end{abstract}

Keywords: thermal barrier coating; thermal vibration experiment; convolutional neural network; feature extraction

\section{Introduction}

Aero engine thermal barrier coating (TBC) is a key thermal protection structure coated on engine turbine blades to ensure reliable operation in high-temperature environments. It is mainly used to reduce the surface temperature of the substrate and resist degradation in various environments [1-4]. TBC materials not only improve the high-temperature resistance and corrosion resistance of the blade material but also largely reduce the generation of defects such as turbine blade cracks, holes, and surface damage, extend the service life of the blade, and can increase the thermal efficiency of the engine by more than 60\% [5]. The performance and service life of blades with TBCs are significantly improved compared to those without TBC, the fuel consumption of aero engines is reduced by $1 \%$ to $2 \%$, and the service temperature of blades is increased by more than $170 \mathrm{~K}$ [6-8]. In general, owing to the harsh service environment, once the TBC peels off and fails, it causes rapid local overheating and burning of metal parts, resulting in aero engine failure and catastrophic consequences. To further utilize the potential of the TBC, maintain the stable thermal insulation performance of the TBC, improve the high-temperature oxidation resistance of the blade, and improve the performance and safety of the engine, the key to ensuring the safe service of the aero engine blade is to study the failure mechanism of the TBC and effectively predict its service life. In particular, the turbine blade as a durability component 
of the aircraft is very expensive. Economic factors should be considered in the actual service. Thus, the research on TBC life prediction has become increasingly important.

The existing TBC interface life analysis method is mainly based on the failure mechanism of the TBC, which is realized by manual formula derivation analysis and ANSYS simulation [9]. Miller, R.A et al. [10] proposed the original TBC life prediction model, which simplified various complex stresses and considered only the radial strain and oxidation factors attributed to the coefficient of thermal expansion. In addition, they developed the National Aeronautics and Space Administration (NASA)'s software Coatlife. Strangman, T. E. et al. [11] further considered the reduced toughness, high-temperature oxidation, and erosion damage of TBC layers in this model. However, the damage of the TBC is a complex process. Courcier, C. et al. [12] improved this method by considering the damage caused by oxide growth and damage caused by thermal cycling and subsequent strain. In such methods, usually, relevant parameters are measured and then used to fit the life. The parameter measurement and formula derivation are complex and inefficient. Jing et al. [13] proposed a TBC life prediction model based on the nonlinear accumulation of oxidative damage and cyclic damage and reported that the error between the damage prediction and test result does not exceed $\pm 10 \%$. Jiang et al. [14] studied the initiation and propagation of cracks at the TBC interface and ceramic layer by cohesive elements and extended finite-element methods. The location and propagation speed of TBC interface cracks have been related to the morphology of the TBC interface and thickness of the TGO (Thermally Growth Oxide). In addition, in an ANSYS simulation, specific conditions need to be limited, and the accuracy is not high. Therefore, the development of an efficient and intelligent coating life prediction technology is needed.

In recent years, image detection algorithms have been widely used in the field of detection. This method has led to numerous achievements in terms of feature classification and scene recognition.

Fu. et al. [15] used three CNN architectures, AlexNet, VGG16, and Resnet34, and improved them to realize bubble detection in defects, with the highest accuracy up to $99.2 \%$. Zhang. et al. [16] extracted features of auxiliary lighting vision sensor system and UVV band vision sensor system through digital image processing and proposed a deep learning algorithm based on $\mathrm{CNN}$ to extract and identify features of three different welding defects in the process of high-power disc laser welding. Wu, Yunzhi et al. [17] used Densenet169 network, Resnet50 network, and MobileNet network for image recognition of multiple plant diseases, and the highest recognition accuracy was $98.97 \%$, which provided a reference for the intelligent diagnosis of plant diseases. Liu, Het al. [18] used CNN for the detection and identification of bridge cracks, and the results showed that the scheme could find all cracks beyond the maximum limit value of bridge cracks, and the recognition rate reached more than $90 \%$, which could provide a reference for intelligent detection of bridge cracks. Elsisi, Mahmoud et al. [19] proposed the IoT architecture based on the machine learning technique for the online monitoring of the gas-insulated switchgear (GIS) status. The results confirm that the technique can visualize all defects in the GIS with different alarms. Elsisi, Mahmoud et al. [20] proposed modified NNA(MNNA) evaluated with the main NNA genetic algorithm-based PID control scheme. The results confirm the robustness and effectiveness of the suggested MNNA-based NLMPC to track regular and irregular trajectories compared with other techniques.

At present, no one has applied the deep-learning image detection algorithm to the intelligent life prediction of thermal barrier coatings. However, the TBC interface topography map contains abundant life information on cracks, gaps, and TGO. The image detection algorithm is used to extract the topographic features. It can be used to analyze the internal correlation mechanism between defects and life, which not only simplifies the complex formula derivation and improves the efficiency of TBC detection, but also promotes deep integration of artificial intelligence and the aviation industry to achieve a reliable TBC detection. Thus, it provides a new theory and method for TBC life prediction. Recently, the team members [21] designed a neural network model based on the VGG-16 model by 
improving the size of the convolution kernel and the moving step size of the convolution kernel, aiming at the complex texture characteristics of the TGO image. Then, the TGO dataset and network structure are used to train the model, and the established model is tested. The accuracy of the improved VGG-16 model is $90.06 \%$ for feature detection of cross-section images of TBCOs collected by different thermal vibration times. However, there are still some problems in the training of TBC images, such as long training time, easy over-fitting, and low accuracy.

Therefore, according to the team's previous work, this paper proposed an Adap-Alex algorithm to overcome the problem originating from traditional convolutional neural networks (CNNs) having too deep structures and too many parameters, which leads to a large training time and over-fitting in training TBC images with complex morphology and structure. The relationship between the TBC interface and life is analyzed. Analyses and design of the thermal vibration experiment related to the TBC life are carried out so that the TBC image characteristics at different thermal vibration times are obtained, and a TBC dataset is constructed. The Adap-Alex algorithm is then used to train the features of the TBC dataset to complete the life detection of the TBC. This study provides a new detection method for TBC life prediction, which is of practical and theoretical significance.

\section{Materials and Methods}

\subsection{Thermal Vibration Experiment}

The thermal vibration experiment is used to simulate the actual service state of a ceramic coating on an aero engine blade. Thermal vibration experiments are commonly used in laboratories to test the performances of TBCs. This experiment also provides a thermal shock test method used to study the oxidation mechanism and failure mechanism of ceramic coatings under high-temperature environmental conditions. The process from the thermal vibration experiment to obtaining the cross-sectional image data of the coating is shown in Figure 1.

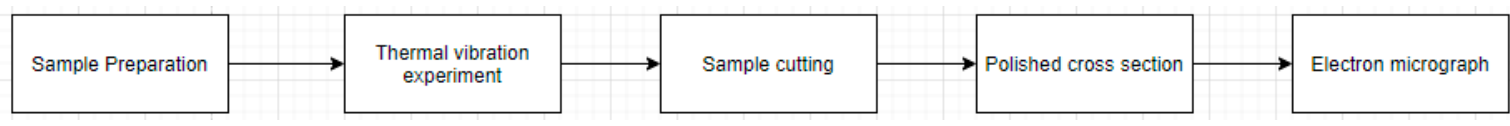

Figure 1. Experiment process.

\subsubsection{Experimental Materials}

In this experiment, atmospheric plasma spraying (APS) technology [22-24] was used to prepare thermal barrier coating samples. The TBC samples were prepared with a nickel-based alloy with a diameter of $50 \mathrm{~mm}$ and a thickness of $10 \mathrm{~mm}$, the bonding layer was 70-80 $\mu \mathrm{m}$, and the surface layer was a YSZ (Yttria-Stabilized Zirconia) coating with a thickness of $210-220 \mu \mathrm{m}$. Totally 300 TBC samples were prepared. Blasting the superalloy substrate with sandblasting machine (Sandblasting air pressure: $0.8 \mathrm{MPa}$; sand specifications: white aluminum oxide: $\mathrm{Al}_{2} \mathrm{O}_{3}$, grain size: $250 \mu \mathrm{m}$; sandblasting strength: $0.7 \mathrm{MPa}$ ). The bonding layer and the ceramic layer were prepared by atmospheric plasma process. Table 1 shows that the process parameters of atmospheric plasma spraying for the ceramic layer and the bonding layer.

Table 1. Spraying parameters of the top coating deposited and bond coating by atmospheric plasma spraying process.

\begin{tabular}{|c|c|c|c|}
\hline Coating & Current/A & Voltage/V & $\begin{array}{l}\text { Powder Flow } \\
\text { Rate } /\left(\mathbf{r} \cdot \min ^{-1}\right)\end{array}$ \\
\hline Bonding layer & 550 & 60 & 2 \\
\hline Surface layer & 600 & 70 & 2 \\
\hline
\end{tabular}


The parameters of the TBC samples are listed in Table 2. The prepared sample of the original TBC is shown in Figure 2.

Table 2. Sample parameters.

\begin{tabular}{|c|c|c|c|c|}
\hline Substrate & Size $/ \mathrm{mm}$ & $\begin{array}{l}\text { Total Coating } \\
\text { Thickness/ } / \mu \mathrm{m}\end{array}$ & Coating Type & $\begin{array}{c}\text { Coating } \\
\text { Thickness/ } \mu \mathrm{m}\end{array}$ \\
\hline $\begin{array}{c}\text { GH635 } \\
\text { Superalloy }\end{array}$ & 50 & $280-300$ & $\begin{array}{l}\text { Bonding layer: } \\
\text { nickel-based } \\
\text { superalloy } \\
\text { Surface layer: } \\
\text { YSZ(Yttria- } \\
\text { Stabilized } \\
\text { Zirconia) }\end{array}$ & $70-80$ \\
\hline
\end{tabular}

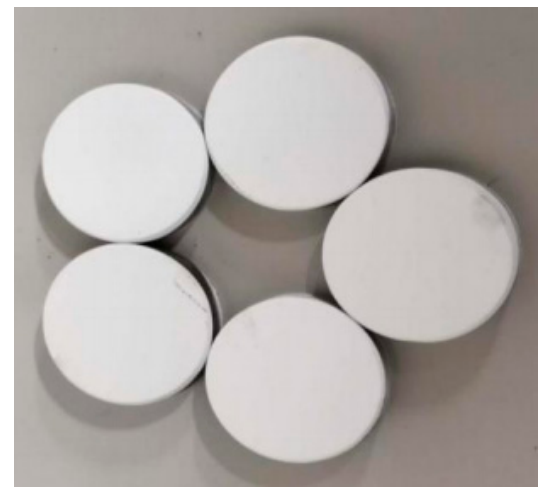

Figure 2. Sample of the TBC.

\subsubsection{Thermal Vibration Experiment Process}

The working temperature of the $\mathrm{TBC}$ was generally above $1050{ }^{\circ} \mathrm{C}$ so that the temperature set in the vibration experiment was $1100^{\circ} \mathrm{C}$. First, the sample was placed in a furnace at $1100{ }^{\circ} \mathrm{C}$, heated for $10 \mathrm{~min}$, and then cooled to room temperature in water. This process was repeated to analyze whether the TBC has cracks, peeling-off, or not.

The interval of the thermal vibrations was set as 60 times; i.e., the number of thermal vibrations in this experiment was 60,120, 180, 240, and 300. In this work, 300 samples were divided into six groups on average, and then the thermal vibration tests were performed.

\subsection{Data Acquisition and Dataset Construction}

\subsubsection{Data Acquisition}

To study the evolution of the internal structure of the TBC, formation of TGO, sintering effect, and phase transformation, it is necessary to cut and polish (Figure 3) the TBC sample after the thermal vibration experiment to obtain the TBC cross-sections. Then, the crosssectional images of the TBC interface obtained with scanning electron microscopy (SEM Figure 4). With the increase in the number of thermal vibrations, the TGO thickness of the TBC interface, content of cracks, and challenges attributed to sintering cracks in the ceramic coating and bonding layer increase significantly. The performances of the TBC interface characteristics for different thermal vibration times are inconsistent. Therefore, the high feature recognition ability of the $\mathrm{CNN}$ can be used to distinguish the image features at different thermal vibration times. The method using CNN to study the TBC is feasible. 


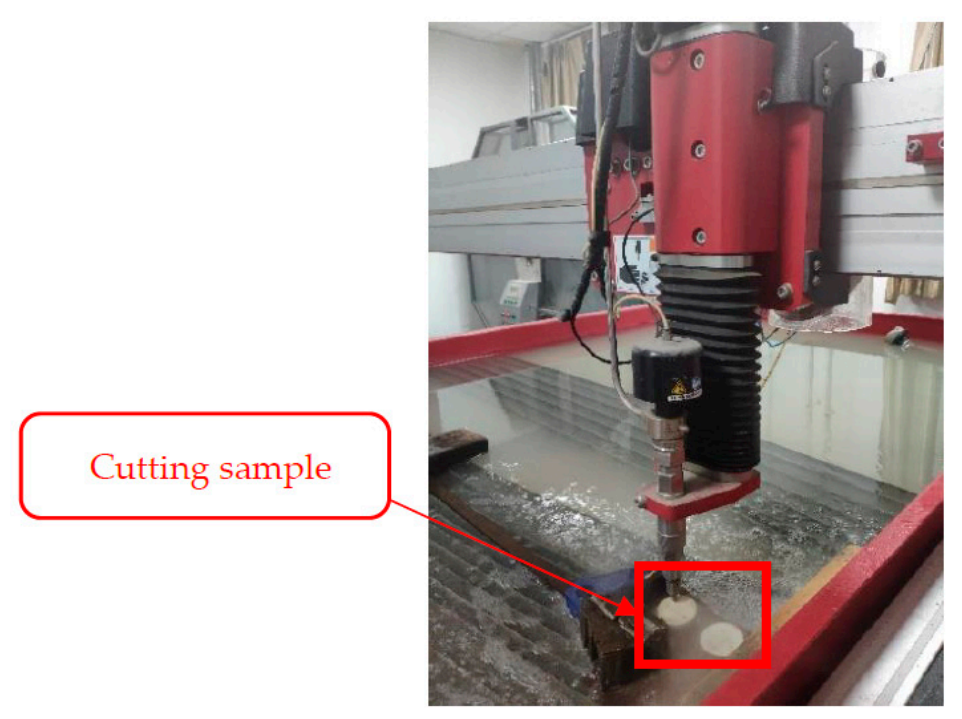

Figure 3. High-pressure waterjet.

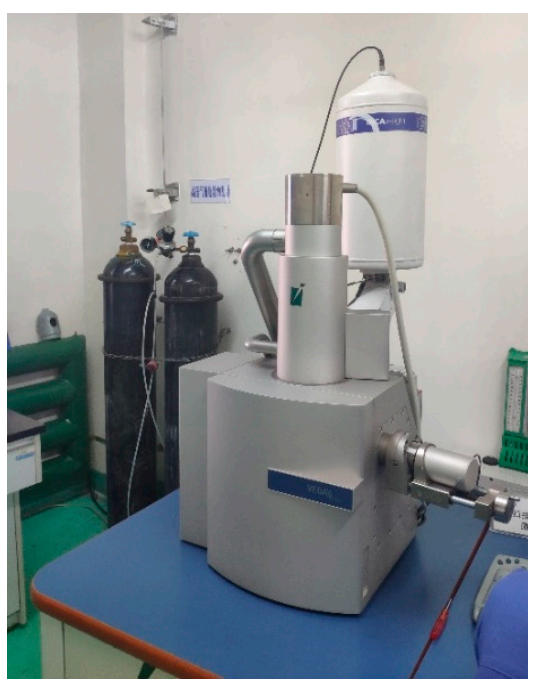

Figure 4. Scanning electron microscope.

\subsubsection{Data Expansion}

After the data are collected, image preprocessing is carried out as an indispensable step in the image detection process to improve the robustness of the model. The preprocessing flow of this experiment is shown in Figure 5.

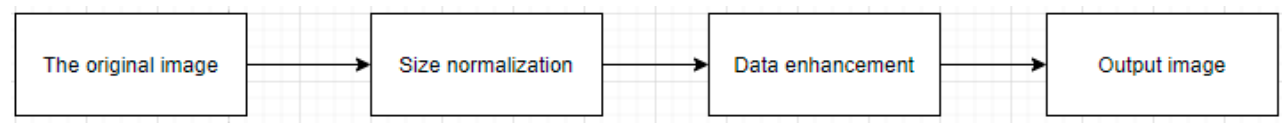

Figure 5. Pretreatment of samples.

To create an image with the same size, it is necessary to normalize the size of the collected original TBC feature images. This experiment uses data enhancement methods such as scaling, random rotation, width offset, height offset, horizontal flip, and mirroring. The operation is completed using the data enhancement function in tensorflow2. The image after the data enhancement is outputted to form the desired sample set. 


\subsubsection{Generation of the Dataset}

The TBC sample set after the data enhancement was divided into six categories based on the number of thermal vibrations $(0,60,120,180,240$, and 300) to obtain the entire TBC data. Partial images of each category are shown in Figure 6.

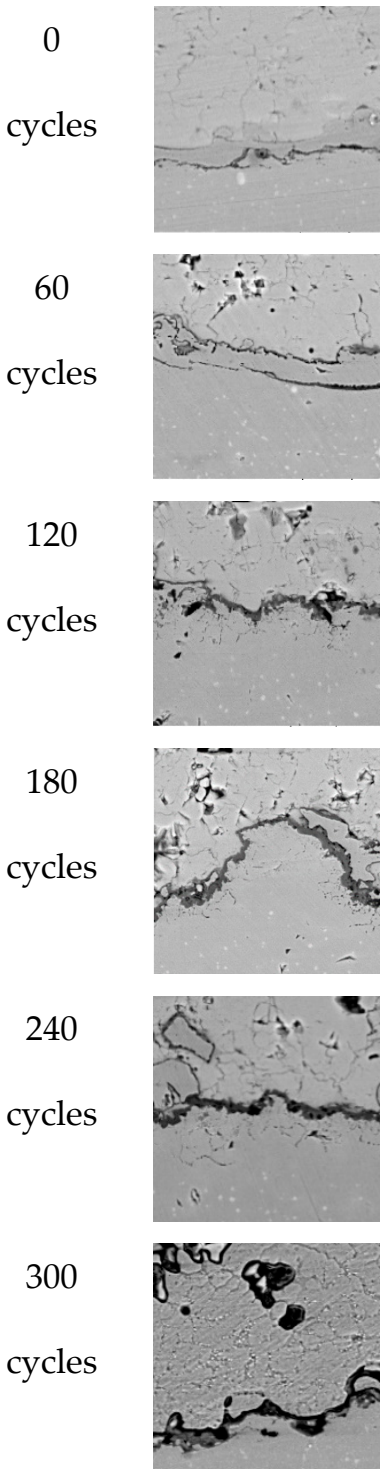

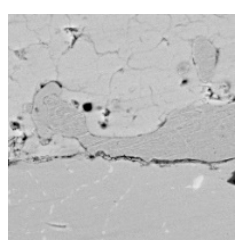
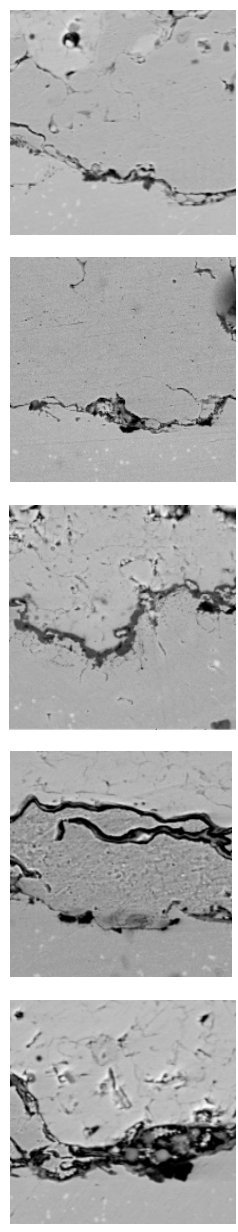

Figure 6. ТВC dataset images.
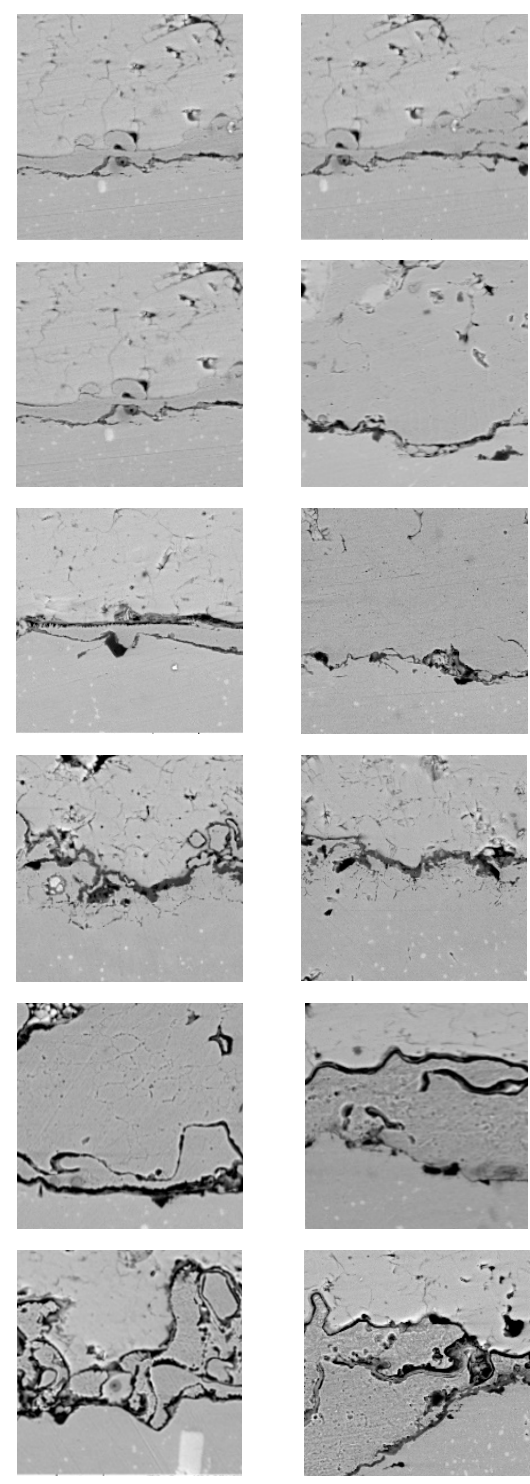

Due to the thermal coating, sample preparation is time-consuming, and thermal vibration experiment is inefficient and time-consuming. Therefore, each category has 50 samples of thermal vibration experiments under the same conditions. After the thermal vibration experiments, 20 images were collected from each sample. In total, 1000 SEM images for each feature category were collected. Usually, image recognition using deep learning requires a large amount of data, and the number of data sets in each category is roughly distributed within the range of 3000 15,000 [15-18]. Hence, the images of each feature category were expanded to 6000 by scaling, random rotation, width offset, height offset, horizontal flipping, mirroring, and other data enhancement methods.

In the creation of the dataset, the feature image labels corresponding to each category of coating are represented by numbers $0,1,2,3,4$, and 5, respectively. Zero represents 0 thermal vibrations, 1 represents 60 thermal vibrations, 2 represents 120 thermal vibrations, 3 represents 180 thermal vibrations, 4 represents 240 thermal vibrations, and 5 represents 
300 thermal vibrations. During training, the dataset is mutually exclusive and divided into a training set and test set. The training set was used to estimate the CNN model, while the test set was used to evaluate the performance of the CNN model. The ratio of the training set to the test set was 4:1; both parts were randomly selected from the sample set.

\subsection{Design and Implementation of the Adap-Alex Algorithm}

The structure of each layer of the proposed Adap-Alex algorithm is presented in Table 3, which shows that the Adap-Alex algorithm uses an eight-layer network, in which the first five layers are convolutional layers. The pool in Table 3 is a pooling operation, not as a separate layer, which is subordinate to the previous convolutional layer. Its function is to effectively reduce the number of parameters. At the same time, adding pooling not only reduces dimensionality, removes redundant information, compresses features, reduces computation, and speeds up computation, but also strengthens the invariance of image features so that it increases the robustness of images in terms of offset and rotation, while the last three layers are fully connected layers. The Adap-Alex algorithm uses a $3 \times 3$ small convolution kernel, which can largely reduce the parameter calculation. Dropout was used on the two layers of Flatten1 and Flatten2 to prevent over-fitting and improve the robustness.

Table 3. Adap-Alex structure parameter setting.

\begin{tabular}{ccccc}
\hline Layer Type & $\begin{array}{c}\text { Receptive Field } \\
\text { Size }\end{array}$ & Stride & BN & Zero Padding \\
\hline Conv1 & $3 \times 3 \times 96$ & 1 & Y & Valid \\
Pool1 & $3 \times 3$ & 2 & - & Valid \\
Conv2 & $3 \times 3 \times 256$ & 1 & - & - \\
Pool2 & $3 \times 3$ & 2 & - & Same \\
Conv3 & $3 \times 3 \times 384$ & 1 & - & Same \\
Conv4 & $3 \times 3 \times 384$ & 1 & - & Same \\
Conv5 & $3 \times 3 \times 256$ & 1 & - & - \\
Pool3 & $3 \times 3$ & - & - & - \\
Flatten1 & 2048 & - & - & - \\
Flatten2 & 2048 & - & - & - \\
Flatten3 & 6 & - & - & - \\
\hline
\end{tabular}

Dropout inhibits a part of the activation unit during training and the activation of a certain neuron stops with a certain probability of $\mathrm{p}$ during forward propagation. This can prevent over-fitting. The neural network model before and after using Dropout is shown in Figure 7.
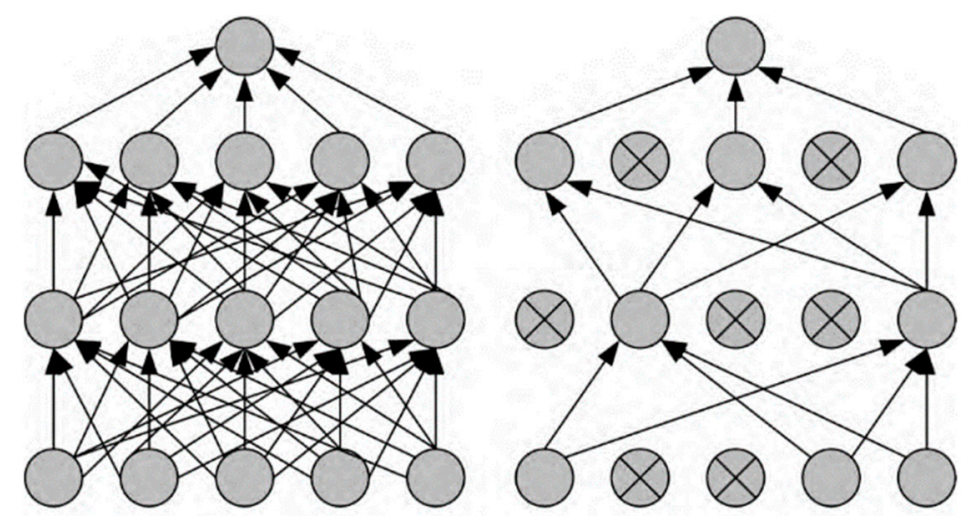

Figure 7. Neural network models before and after using Dropout.

Pooling layers are mainly used to reduce the amount of feature data and calculation. Two main methods are used: max pooling, which uses the maximum value in the sliding 
window frame as an output, which provides the advantage of extracting image texture, and mean pooling, which uses the average value of all data in the sliding window frame as an output, which has the advantage of retaining the background features.

Figure 8 shows that the maximum pooling considers only the maximum value of the sliding window. If the maximum value in the sliding window is considerably larger than that of the other elements, other features will disappear after the maximum pooling operation. Mean pooling calculates the average value of all elements in the sliding window area. Although all values are considered, this operation will blur more important features. Therefore, the Adap-Alex algorithm overcomes the problem originating from the traditional pooling method that cannot extract effective feature values. According to the combination of the number of training epochs of the network and sigmoid function, the mixture ratio of the classical pooling is adjusted.
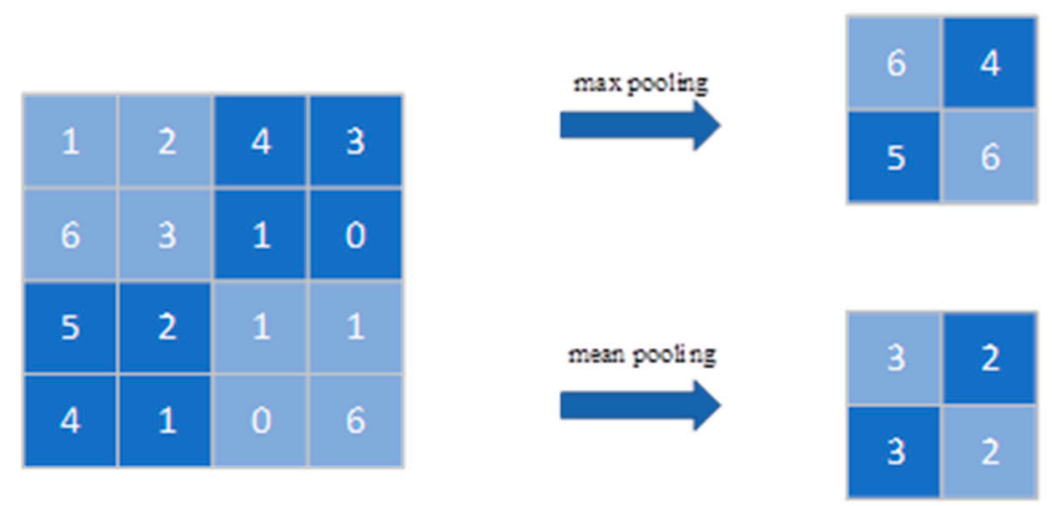

Figure 8. Max pooling and mean pooling.

The sigmoid function is often used to construct an artificial neural network (ANN) activation function in neural networks. It provides a good balance between linearity and nonlinearity $[25,26]$.

The Adap-Alex algorithm combines the sigmoid function with the number of training epochs of the network to determine the mixing ratio of the maximum pooling and average pooling. The specific improvement operation is expressed by

$$
P_{A P}=\frac{P_{\max }-P_{a v c}}{1+\exp \left(\frac{n_{e p o c h}}{2}-n_{t}\right)}+P_{a v c}
$$

where $P_{A P}$ is the adaptive pooling output, $P_{\max }$ is the maximum pooling result, $P_{\max }(X)=$ $\max \left(X_{i}\right), P_{a v c}$ is the average pooling result, $P_{a v c}=\frac{1}{N} \sum_{i=1}^{N} X_{i}, n_{\text {epoch }}$ is the number of rounds of network training, and $n_{t}$ is the network training time.

The formula shows that $P_{A P}$ changes with $n_{t}$, as presented in Figure 9.

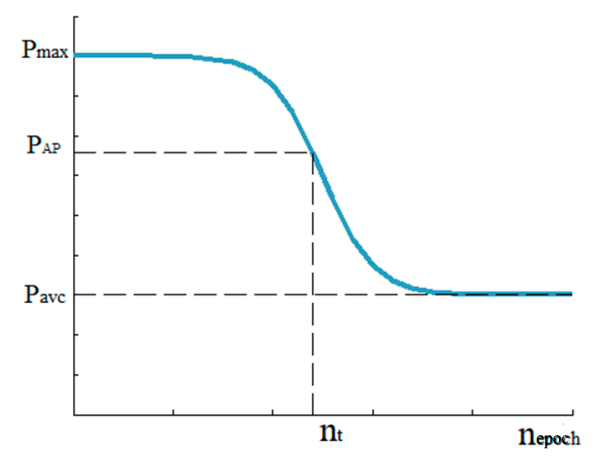

Figure 9. Improved adaptive tuning curve. 
In the initial stage of neural network training, the probability of maximum pooling should be increased, the probability of average pooling should be reduced, and the texture features of the picture should be fully extracted. In the mid-training period, the maximum pooling and average pooling probabilities adaptively change according to the sigmoid curve with the number of training rounds. In the later stage of neural network training, the probability of average pooling should be increased, the probability of maximum pooling should be reduced, and the overall data characteristics should be fully retained. The most significant effect of the adaptive pooling output is to improve the accuracy of the model and learning ability of the network with the mixing ratio between the maximum value and average value.

In training, the loss function is typically used as an index to evaluate the quality of the parameters. The cross-entropy loss function can be used to calculate the difference between the predicted value of the model and true value. A smaller value implies a closer distribution of the two values and higher performance of the model. A larger value indicates that the model is unstable and needs to be further improved. The cross-entropy loss function is defined as

$$
\text { loss }=-\sum_{i=1}^{n} y_{i} \log \left(y_{\mathrm{i}}\right)
$$

where $y_{i}$ is the true value of the data, $y_{i}$ is the predicted value of the neural network, and $n$ is the number of samples.

To obtain the optimal weight parameters of the network, an optimization method was used to optimize the loss function to obtain the minimum loss. When the loss function is smallest, the network optimal parameter update is achieved through the gradient information of the network backpropagation. Adaptive moment estimation (Adam) [27] is a parameter adaptive learning rate method based on first-order moment estimation and second-order moment estimation of the gradient to dynamically adjust the learning rate of each parameter, expressed by

$$
\begin{gathered}
g_{\theta}=\nabla_{\theta} \sum L\left(f\left(x^{(i)} ; \theta\right), y^{(i)}\right) \\
d_{i}=\beta_{1} d_{i-1}+\left(1-\beta_{1}\right) g_{\theta} \\
S_{i}=\beta_{2} S_{i-1}+\left(1-\beta_{2}\right) g_{\theta}^{2} \\
\hat{d}_{i}=\frac{d_{i}}{1-\beta_{1}^{i}} \\
\hat{S}_{i}=\frac{S_{i}}{1-\beta_{2}^{i}} \\
\theta_{i} \leftarrow \theta_{i-1}-\frac{\alpha}{\sqrt{\hat{S}_{i}+\varepsilon}} \hat{d}_{i}
\end{gathered}
$$

where $L$ is the loss function, $x^{(i)}$ are the training samples, $\alpha$ is the learning rate, $\hat{d}_{i}$ is the bias correction of $d_{i}, \hat{S}_{i}$ is the bias correction of $S_{i}, \beta_{1}, \beta_{2}$ are empirical parameters (usually $\beta_{1}$ is 0.9 and $\beta_{2}$ is 0.999 ), and $\varepsilon$ is $10^{-8}$. $\hat{d}_{i}$ and $\hat{S}_{i}$ are used to adjust the previous error, which can be approximated as an unbiased estimate of the expectation.

The other training parameters of the model are presented in Table 4.

Table 4. Model training parameters.

\begin{tabular}{cc}
\hline Parameter & Numerical Value \\
\hline Batch size & 64 \\
Iteration number & 100 \\
Dropout & 0.5 \\
\hline
\end{tabular}

\section{Results}

\subsection{Image Analysis Results}

The samples were divided into six groups according to the frequency of thermal vibration from 0 to $60,120,180,240$, and 300 . In the algorithm model classification, according to the TGO thickness after the different number of thermal vibration experiments 
of the sample, the classification results are divided into three categories: safe, critical, and damaged; and identify the thermal vibration times of the thermal barrier coating according to the TGO thickness interval. When the number of thermal vibrations is less than or equal to 180 times, the thickness of all TGO is less than $8 \mu \mathrm{m}$, which is classified as safe. When the number of thermal vibrations is 240 times, the thickness of most TGO samples was close to $8 \mu \mathrm{m}$, which is in a critical state. When the number of thermal vibrations is 300 times, almost all TGO samples are greater than $8 \mu \mathrm{m}$, and the thermal barrier coating is in a failure state [28-33]. The statistical table of TGO thickness of samples with the increase of thermal vibration times and its distribution interval are shown in Table 5. Figure 10 shows the images of the coating in Security, Critical and Damage states respectively.

Table 5. Statistical table of test thickness of some samples of SEM with different times of thermal vibration (unit: $\mu \mathrm{m}$ ).

\begin{tabular}{cccc}
\hline & $\begin{array}{c}\text { Scanning Electron } \\
\text { Microscope Image of } \\
\text { the Original Sample }\end{array}$ & $\mathbf{6 0 \text { Cycles }}$ & $\mathbf{1 2 0}$ Cycles \\
\hline $\operatorname{Min}$ & 0.427 & 0.607 & 1.537 \\
$\operatorname{Max}$ & 0.596 & 1.432 & 1.988 \\
$\operatorname{Min}$ & 180 cycles & 240 cycles & 300 cycles \\
$\operatorname{Max}$ & 2.091 & 5.621 & 7.887 \\
& 5.432 & 7.695 & 12.432 \\
\hline
\end{tabular}

Security
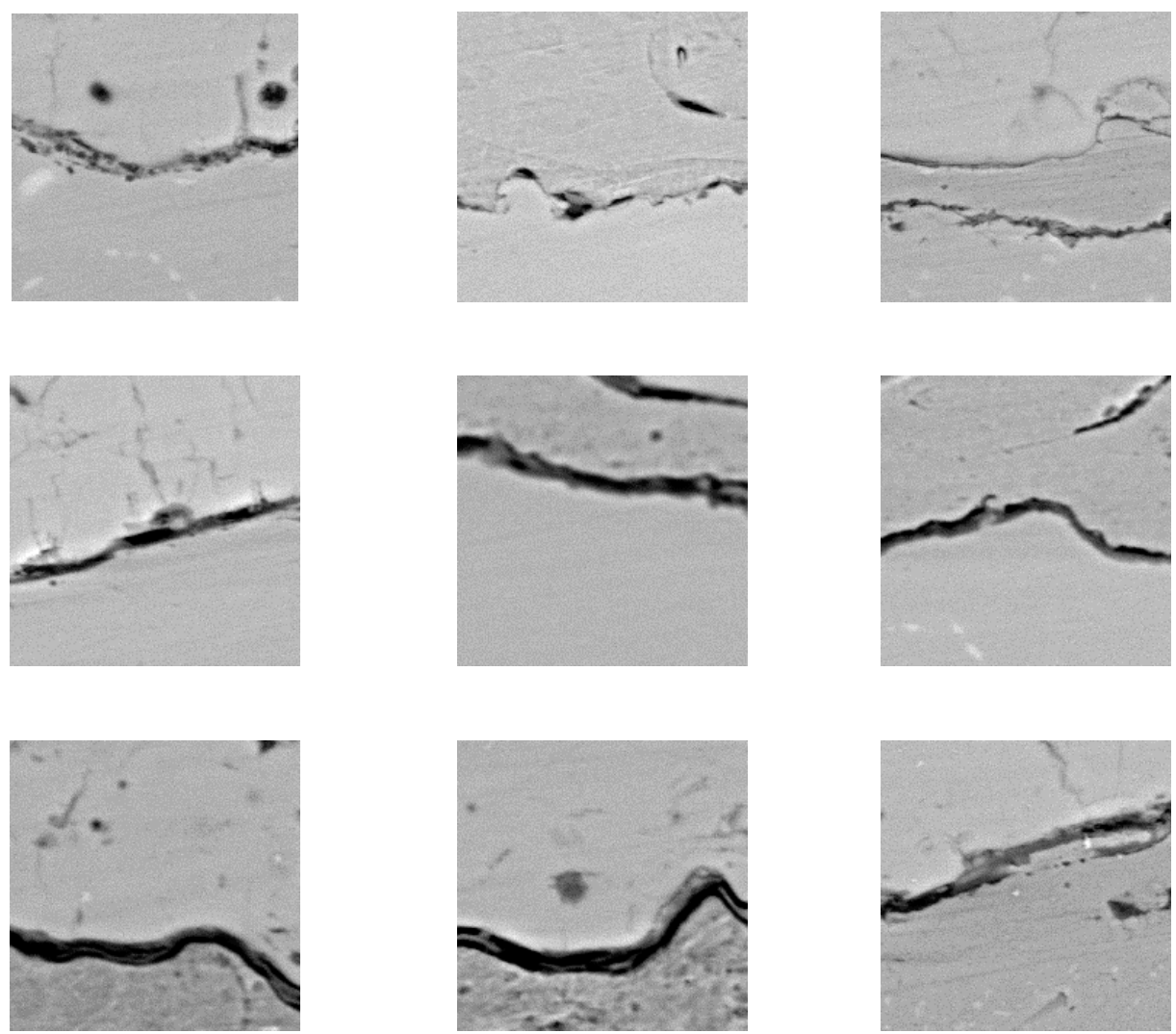

Figure 10. Three random images of each category in TGO dataset.

\subsection{Algorithm Recognition Results}

To verify the performance of the Adap-Alex method designed in this study, VGGNet16 and Alex-Net were used to train and compare the six types of TBC feature training sets; the other parameter settings were consistent. The training times of each iteration are 
compared in Table 6, while the accuracy and loss curve of each iteration during the training are shown in Figure 11.

Table 6. Training time comparison.

\begin{tabular}{cc}
\hline Algorithm & Time/s \\
\hline VGG-Net & 1054 \\
Alex-Net & 1614 \\
Adap-Alex & 929 \\
\hline
\end{tabular}

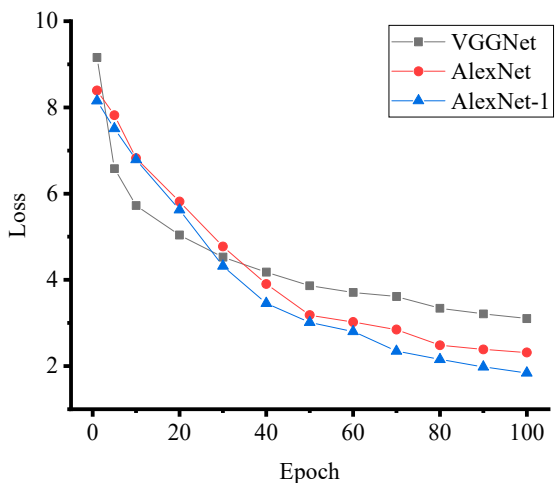

(a) Training accuracy

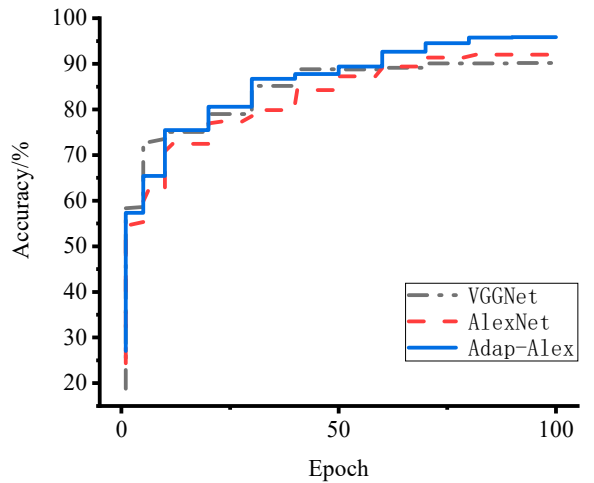

(b) Loss curve

Figure 11. Training accuracy and loss curve.

As shown in Table 6, the training time of the Adap-Alex algorithm is significantly smaller; $125 \mathrm{~s}$ smaller than that of VGG-Net and $685 \mathrm{~s}$ smaller than that of Alex-Net. Figure 11a shows that, although the VGG-Net structure converges quickly, the model tends to converge after approximately 30 trainings, but its final accuracy is lower than that of the Alex-Net structure. The iterative convergence speeds of Alex-Net and the improved Adap-Alex structure are not significantly different. The model tends to converge after approximately 80 iterations, but the final accuracy of Adap-Alex is highest. As shown in Figure 11b, the loss value of the Adap-Alex structure is relatively small in the later training stage, indicating that the improved Adap-Alex structure network model is relatively stable in the later training stage and that the performance of the model is higher.

After the training with VGG-Net, Alex-Net, and Adap-Alex, the test accuracies for the six TBC thermal vibration times are shown in Table 7.

Table 7. Recognition accuracies of the three network structures.

\begin{tabular}{ccccccc}
\hline \multirow{2}{*}{ Algorithm } & \multicolumn{6}{c}{ Accuracy/\% } \\
\cline { 2 - 7 } & 0 Cycles & 60 Cycles & 120 Cycles & $\mathbf{1 8 0}$ Cycles & 240 Cycles & 300 Cycles \\
\hline VGG-Net & 73 & 72 & 74 & 83 & 81 & 88 \\
Alex-Net & 75 & 73 & 80 & 85 & 88 & 90 \\
Adap- & 85 & 84 & 88 & 87 & 92 & 93 \\
Alex & & & & &
\end{tabular}

As shown in Table 7, the test accuracy of VGG-Net for 300 cycles of thermal vibration is $88 \%$, which is $15 \%, 16 \%, 14 \%, 5 \%$, and $7 \%$ higher than the accuracies at $0,60,120,180$, and 240 cycles of thermal vibration, respectively. Alex-Net has a recognition accuracy rate of $90 \%$ at 300 thermal vibrations, $15 \%, 17 \%, 10 \%, 5 \%$, and $2 \%$ higher than those at $0,60,120$, 180 , and 240 thermal vibrations, respectively. The recognition accuracy rate of Adap-Alex at 300 thermal vibrations is $93 \%$, which is $8 \%, 9 \%, 5 \%, 6 \%$, and $1 \%$ higher than those at 0 , $60,120,180$, and 240 thermal vibrations, respectively. 


\section{Discussion}

According to the data, when the number of thermal vibrations is 0 , the test accuracy of Adap-Alex is $85 \%, 12 \%$, and $10 \%$ higher than those of VGG-Net and Alex-Net, respectively. When the number of thermal vibrations is 60 , the test accuracy of Adap-Alex is $84 \%, 12 \%$, and $11 \%$ higher than those of VGG-Net and Alex-Net, respectively. When the number of thermal vibrations is 120, the test accuracy of Adap-Alex is $88 \%, 14 \%$, and $8 \%$ higher than those of VGG-Net and Alex-Net, respectively. When the number of thermal vibrations is 180 , the test accuracy of Adap-Alex is 87\%, 4\%, and 2\% higher than those of VGG-Net and Alex-Net, respectively. When the number of thermal vibrations is 240 , the test accuracy rate of Adap-Alex is $92 \%, 11 \%$, and $4 \%$ higher than those of VGG-Net and Alex-Net, respectively. When the number of thermal vibrations is 300, the test accuracy of Adap-Alex is $93 \%, 5 \%$, and 3\% higher than those of VGG-Net and Alex-Net, respectively. The above analysis shows that, for the same algorithm, a higher number of thermal vibrations led to higher test accuracy, since more cracks appeared in the coating, the sintering was more severe, the TGO layer was thicker, and more features were exhibited by the TBC, which facilitated the detection. When the number of thermal vibrations is constant, the accuracy of Adap-Alex is relatively high. Therefore, it can more easily identify the coating than the other two algorithms. The overall test accuracy of VGG-Net is low; the highest value of $88 \%$ is obtained when the number of thermal vibrations is 300 . The test accuracy of Alex-Net is higher than that of VGG-Net; its highest accuracy of $90 \%$ is obtained when the number of thermal vibrations is 300 . The designed Adap-Alex algorithm has a higher test accuracy rate than those of the other two structures; its highest accuracy rate of $93 \%$ was obtained at 240 thermal vibrations. Thus, the Adap-Alex algorithm designed in this study has not only a shorter training time period but also largely improved accuracy. Overall, the comparison of the results of the MNIST and CIFAR-10 datasets shows that the recognition accuracy for the TBC and convergence speed are relatively low. Thus, the quality and size of the developed TBC dataset need to be improved.

\section{Summary of Results}

The experimental results and the algorithm recognition results are summarized as follows:

(1) When the number of thermal vibrations is below 180 times, the thickness of all TGO is less than $8 \mu \mathrm{m}$, which is classified as safe. As for when the number of thermal vibrations equals 240 times, the thickness of most TGO samples was close to $8 \mu \mathrm{m}$, indicating a critical state. While the number of thermal vibrations is over 300 times, almost all TGO samples are greater than $8 \mu \mathrm{m}$, and the thermal barrier coating is in a failure state.

(2) TBC data were obtained through thermal vibration experiments and processed (size normalization, data expansion), and then a TBC dataset was constructed. The improved algorithm was used to verify its effectiveness on the generated TBC dataset. The Adap-Alex algorithm training time was significantly smaller, $125 \mathrm{~s}$ smaller than that of VGG-Net and $685 \mathrm{~s}$ smaller than that of Alex-Net. When the number of thermal vibrations was fixed, the Adap-Alex test accuracy was higher than those of VGG-Net and Alex-Net, which facilitated the identification of the characteristics of the coating. The best result was achieved when the number of thermal vibrations was 300; the accuracy rate reached $93 \%$. This shows that the algorithm proposed in this paper provides better learning and can more easily predict the life of the TBC.

The following future works can be conducted:

In the part of thermal barrier coating data acquisition, some kinds of thermal barrier coatings can be studied according to different experimental conditions, i.e., thermal shock times and environments, etc. More researches can be conducted by changing TBC materials in order to enrich models of the algorithm. Serious thermal vibration situations can be considered to prevent over-fitting and improve the accuracy of TBC identification effectively. 


\section{Conclusions}

In this paper, deep learning is introduced into the TBC life prediction research for improving the inefficiency of the TBC life prediction research method. An Adap-Alex algorithm was proposed and verified by experiments. The following conclusions are reached:

(1) On the basis of retaining the image features to the maximum extent, the parameter calculation can be greatly reduced by using a small convolution kernel in the structure. Adap-Alex adopts a small convolution kernel with the size of $3 \times 3$, and the moving step size of 1. Compared with Alex-Net and VGG-16, the network training time of Adap-Alex is significantly reduced by $42.44 \%$ and $11.86 \%$.

(2) Optimal pooling can greatly improve the ability of neural networks to learn features. The mixing ratio of classical pooling was adjusted through the Sigmoid function in the pooling method. Compared with the traditional pooling method, this adaptive pooling method combines the advantages of maxi-mum pooling and average pooling extraction features so that important features are retained and unimportant features are discarded.

Author Contributions: R.G. and W.M. conceived and designed the experiments. W.M., Y.W., and S.F. performed the experiments. W.S. improved the experimental plan and guided the writing and revision of the article. W.M., S.F., and Y.W. completed the data analysis and the writing of the first draft of the article. All authors have read and agreed to the published version of the manuscript.

Funding: This research was funded by the National Natural Science Foundation of China, No. 51775433, the Key Research and Development Program of Shaanxi Province of China (Grant No. 2021GY-115), and the Key Research and Development Program of Shaanxi Province of China (Grant No. 2021GY-260), Natural Science Basic Research Program of Shaanxi (Program No.2021JQ-049); China Postdoctoral Science Foundation funded project (No.2021M692516).

Institutional Review Board Statement: Not applicable.

Informed Consent Statement: Not applicable.

Data Availability Statement: Data is contained within the article.

Conflicts of Interest: The authors declare no conflict of interest.

\section{References}

1. Cao, X.Q.; Vassen, R.; Stoever, D. Ceramic materials for thermal barrier coatings. J. Eur. Ceram. Soc. 2004, 24, 1-10. [CrossRef]

2. Gao, Y. Brief introduction and trend exploration of aero engine development. Mod. Manuf. Technol. Equip. $2018,7,222$.

3. Yang, X.; Geng, R.; Zhou, Y. Research on Thermal Fatigue Life Prediction Method of Thermal Barrier Coating. J. Aerosp. Power 2003, 18, 201-205.

4. Sun, J.; Xu, Y.; Li, W.; Chen, Y.; Lv, K. Residual Stress and Failure Analysis of Thermal Barrier Coating under Thermal Growth. J. China Surf. Eng. 2016, 29, 25-31.

5. Song, J.; Li, S.; Qi, H.; Shi, D.; Yang, X. Research Progress on Failure Behavior and Life Prediction of Thermal Barrier Coatings. J. Aviat. Manuf. Technol. 2019, 62, 34-40.

6. Mou, R.; Lu, F.; He, L.; He, S.; Huang, G. Application and Development of Thermal Barrier Coating Technology on Aero-Engine. J. Thermal Spray Technology. 2009, 1, 1.

7. Zhao, T. The Necessity Analysis of Turbofan Engine Thrust Reduced Takeoff. J. Civ. Aviat. Univ. China. 2005, 23, 7-9.

8. Padture, N.P. Thermal Barrier Coatings for Gas-Turbine Engine Applications. Science 2002, 296, 280-284. [CrossRef]

9. Tang, J.; Li, Z. Stress Analysis of Plasma Coating on Turbine Blade. J. Proj. Rocket Guid. 2010, 30, $227-229$.

10. Miller, R.A. Oxidation-based model for thermal barrier coating life. J. Am. Ceram. Soc. 2010, 67, 517-521. [CrossRef]

11. Strangman, T.E.; Neumann, J.F.; Liu, A. Thermal barrier coating life prediction model development. Surf. Coat. Technol. 1987, 32, 305-306.

12. Courcier, C.; Maurel, V.; Rémy, L.; Quilici, S.; Rouzou, I.; Phelippeau, A. Interfacial damage based life model for EB-PVD thermal barrier coating. Surf. Coat. Technol. 2011, 205, 3763-3773. [CrossRef]

13. Huang, J.; Wang, W.; Lu, X.; Liu, S.; Li, C. Influence of lamellar interface morphology on cracking resistance of plasma-sprayed ysz coatings. Coatings 2018, 8, 187. [CrossRef]

14. Jing, F.; Yang, J.; Yang, Z.; Zeng, W. Critical compressive strain and interfacial damage evolution of EB-PVD thermal barrier coating. Mater. Sci. Eng. A 2020, 776, 139038. [CrossRef]

15. Fu, Y.; Aldrich, C. Flotation froth image recognition with convolutional neural networks. Miner. Eng. 2019, 132, 183-190. [CrossRef] 
16. Zhang, Y.; You, D.; Gao, X. Welding defects detection based on deep learning with multiple optical sensors during disk laser welding of thick plates. J. Manuf. Syst. 2019, 51, 87-94. [CrossRef]

17. Wu, Y.; Liu, A.; Zhu, X.; Liu, C.; Fan, G.; Le, Y.; Zhang, Y. A convolutional network architecture for plant disease image recognition. J. Anhui Agric. Univ. 2021, 1-7.

18. Liu, H.; Wang, X.; Li, B.; Meng, J. Detection and recognition of bridge crack based on convolutional neural network. J. Hebei Univ. Sci. Technol. 2016, 37, 485-490.

19. Elsisi, M.; Tran, M.Q.; Mahmoud, K.; Mansour, D.-E.A.; Lehtonen, M.; Darwish, M.M.F. Towards Secure Online Monitoring for Digitalized GIS against Cyber-Attacks based on IoT and Machine Learning. IEEE Access 2021, 9, 78415-78427. [CrossRef]

20. Elsisi, M.; Mahmoud, K.; Lehtonen, M.; Darwish, M.M.F. Effective Nonlinear Model Predictive Control Scheme Tuned by Improved NN for Robotic Manipulators. IEEE Access 2021, 9, 64278-64290. [CrossRef]

21. Zhao, L. Intelligent Identification Method for Characteristics of Thermally Groen Oxide Layer. Master's Thesis, Xi'an University of Technology, Xi'an, China, 2020.

22. Fan, J.; Li, X.; Zhang, B.; Chu, Y. Growth and evolution of thermally grown oxides in thermal barrier coating produced by thermal spraying. Heat Treat. Met. 2018, 43, 2.

23. Peng, X. The Evaluation of Microstructure Regulation and Mechanism of the Double-Layer Bond Coat in Thermal Barrier Coatings. Master's Thesis, Xi'an Shiyou University, Xi'an, China, 2019.

24. Du, S.; Liu, C.; Cai, H.; Huan, Q.; Zhang, Y. Preparation and tribological properties of plasmaSprayed $\mathrm{Cu}_{-} \mathrm{Al}_{2} \mathrm{O}_{3}$ composite coatings. J. Surface Technol. 2019, 48,3.

25. Yonaba, H.; Anctil, F.; Fortin, V. Comparing Sigmoid Transfer Functions for Neural Network Multistep Ahead Streamflow Forecasting. J. Hydrol. Eng. 2010, 15, 275-283. [CrossRef]

26. Gao, R.; Fan, S. Research on the Propagation Characteristics of Fatigue Cracks on Rail Surfaces. Int. J. Appl. Mech. 2020, 12, 10. [CrossRef]

27. Luo, Z.; Chen, Y.; Li, C.; Xiong, X.; Zhu, L. Minimum BER Criterion and Adaptive Moment Estimation Based Enhanced ICA for Wireless Communications. IEEE Access 2020, 8, 152071-152080. [CrossRef]

28. Wang, L. Study Oninfluence of Morphology and Thickness of TGO on Failure Mechanism of Thermal Barrier Coating. Master's Thesis, Northwestern Polytechnical University, Xi'an, China, 2015.

29. Miao, W.; Wang, L.; Guo, H.; Peng, H.; Wang, K.; Gong, S. Thermal cycling behavior and associated failure mechanism of EB-PVD thermal barrier coatings with CMAS deposits. Acta Mater. Compos. Sin. 2012, 5, 76-82.

30. Jiang, J.; Wang, W.; Zhao, X.; Liu, Y.; Cao, Z.; Xiao, P. Numerical analyses of the residual stress and top coat cracking behavior in thermal barrier coatings under cyclic thermal loading. Eng. Fract. Mech. 2018, 196, 191-205. [CrossRef]

31. Li, Y.; Huang, J.; Wang, W.; Ye, D.; Fang, H.; Gao, D.; Tu, S.; Guo, X.; Yu, Z. Control of the Pore Structure of Plasma-Sprayed Thermal Barrier Coatings through the Addition of Unmelted Porous YSZ Particles. Coatings 2021, 11, 360. [CrossRef]

32. Mauer, G.; Du, L.; Vaßen, R. Atmospheric Plasma Spraying of Single Phase Lanthanum Zirconate Thermal Barrier Coatings with Optimized Porosity. Coatings 2016, 6, 49. [CrossRef]

33. Liu, E.; Zhang, J.; Chen, S.; Du, S.; Du, H.; Cai, H.; Wang, L. High temperature negative wear behaviour of VN/Ag composities induced by expansive oxidation reaction. Ceram. Int. 2021, 47, 15901-15909. [CrossRef] 